\title{
Stabilization of a certain class of fuzzy control systems with uncertainties
}

\author{
NIZAR HADJ TAIEB, MOHAMED ALI HAMMAMI and FRANÇOIS DELMOTTE
}

\begin{abstract}
In this paper, we investigate the global uniform practical exponential stability for a class of uncertain Takagi-Sugeno fuzzy systems. The uncertainties are supposed uniformly to be bounded by some known integrable functions to obtain an exponential convergence toward a neighborhood of the origin. Therefore, we use common quadratic Lyapunov function (CQLF) and parallel distributed compensation (PDC) controller techniques to show the global uniform practical exponential stability of the closed-loop system. Numeric simulations are given to validate the proposed approach.
\end{abstract}

Key words: Takagi-Sugeno fuzzy systems, PDC controller, global uniform practical exponential stability, Lyapunov stability, parametric uncertainty.

\section{Introduction}

It is well known that most plants in industry show significant nonlinearities, which usually make the analysis and controller design difficult. In order to overcome such difficulties, various schemes have been developed in the past two decades, among which a successful approach is the fuzzy control ( [20], [22], [23], [30]). In recent years, TakagiSugeno (T-S) fuzzy models [22] have become a useful tool to deal with a class of nonlinear systems. The models can be described by a set of "if-then" rules which gives local linear approximations of an underlying system.

The stability analysis and control design for T-S fuzzy systems keep attracting researchers for decades ( [1], [6], [7], [26], [27], [31]). The Lyapunov stability theory is the main approach for these kinds of problems. Among them, the simplest approaches consists in looking for a common quadratic Lyapunov function (CQLF) by using the concept of the parallel distributed compensation (PDC) technique ( [19], [26], [27], [29]) to design a stabilizing controller. However, another important issue in stability analysis of nonlinear systems may be how to study the behavior of the solutions in the case when

N.H. Taieb and M.A. Hammami are with University of Sfax, Tunisia, Faculty of Sciences of Sfax, Department of Mathematics. E-mails: Nizar.HadjTaieb@yahoo.fr, MohamedAli.Hammami@ fss.rnu.tn. F. Delmotte is with University of Artois, France, e-mail: francois.delmotte@ univ-artois.fr. Corresponding author is M.A. Hammani.

Received 6.3.2017. 
they converge to a small neighborhood of the origin. To deal with these situation, the concept of practical stability ( [12], [13], [16]), which is derived from the so called, finite time stability, is more useful. Indeed, the practical stability for nonlinear systems has been widely investigated in mathematical theory. In these studies, the origin was not supposed to be an equilibrium point of the system. So, we can no longer expect to design a controller that guarantees the stability of the origin as an equilibrium point. In [3], [17] and [18], some controllers are constructed to guarantee exponential stability of a ball containing the origin of the state space where the radius of this ball can be made arbitrary small. The authors in [33], introduced the notion of input to state practical stability to design of robust adaptive controllers for nonlinear systems with dynamic uncertainties. In [32], the concept of input to state practical stability is extended to stochastic case and an output feedback controller is proposed for a class of stochastic nonlinear systems with uncertain nonlinear functions. By using the fuzzy approach the authors in [14], have investigated the practical stability of a class of uncertain T-S fuzzy systems where the uncertainties satisfy the so called matching conditions.

In this paper, we deal with the uniform ultimate boundedness for a class of TakagiSugeno fuzzy systems in presence of external disturbances. The objective is to guarantee, no matter how we select the uncertain external disturbances, that the state will eventually end up and remain within some pre-specified region. When this region is a small neighborhood about the origin, the concept of uniform ultimate boundedness is equivalent to practical stability. Therefore, we are interested in studying the global uniform practical exponential stability for a class of uncertain Takagi-Sugeno fuzzy systems in term of convergence toward a neighborhood of the origin. The main novelty of this paper relies on the fact that the proposed approach for stability analysis allows for the computation of the bound which characterize the exponential rate of convergence of the solutions. The common quadratic Lyapunov function and parallel distributed compensation controller are used to show the ultimate boundedness of the solutions of the uncertain T-S fuzzy systems, even when the origin is not an equilibrium point of the system, provided that the uncertainties are supposed uniformly bounded by known integrable functions. Compared to classical LMIs conditions, the new LMIs are a little bit more severe in order to handle the uncertainties. Then, it is possible to prove systems performance by adjusting the practical stability conditions.

The remainder of this paper is organized as follows: section 2 reviews the conventional T-S fuzzy model and issues about stability. Section 3 presents the global uniform practical exponential stability for T-S fuzzy uncertain systems in term of convergence toward a neighborhood of the origin, furthermore new LMIs are presented in order to handel the uncertainties. Section 4 presents the numerical examples. 


\section{Takagi-Sugeno fuzzy systems}

Consider a class of the continuous-time T-S fuzzy control system which can be described by the following fuzzy rules,

Rule $i:$ If $z_{1}(t)$ is $M_{i 1}$ and $z_{2}(t)$ is $M_{i 2} \ldots$ and $z_{p}(t)$ is $M_{i p}$, then

$$
\dot{x}(t)=A_{i} x(t)+B_{i} u(t), i=1,2, \ldots, r,
$$

where $x(t) \in \mathbb{R}^{n}$ is the state vector, $u(t) \in \mathbb{R}^{m}$ is the control input vector, $A_{i} \in \mathbb{R}^{n \times n}$ and $B_{i} \in \mathbb{R}^{n \times m}$ are the system matrix input matrix, $i=1, \ldots, r$ is the number of fuzzy rules, $M_{i j}$ are the inputs fuzzy sets, $z(t)=\left[z_{1}(t), \ldots, z_{p}(t)\right]^{T}$ are measurable variables, i.e., the premise variables. Using weighted average defuzzifiers, the aggregated fuzzy model is given by

$$
\dot{x}(t)=\frac{\sum_{i=1}^{r} w_{i}(z)\left(A_{i} x(t)+B_{i} u(t)\right)}{\sum_{i=1}^{r} w_{i}(z)},
$$

where

$$
w_{i}(z)=\prod_{i=1}^{r} M_{i j}\left(z_{j}\right)
$$

Let $\mu_{i}(z)$ be the membership functions that belong to class $C^{1}$, i,e., they are continuous differentiable and defined as

$$
\mu_{i}(z)=\frac{w_{i}(z)}{\sum_{i=1}^{r} w_{i}(z)} .
$$

Then the fuzzy system has the state-space form

$$
\dot{x}(t)=\sum_{i=1}^{r} \mu_{i}(z)\left(A_{i} x(t)+B_{i} u(t)\right) .
$$

$\mu_{i}$ are such that $\mu_{i}(z) \geqslant 0$ for $i=1,2, \ldots, r$ and $\sum_{i=1}^{r} \mu_{i}(z)=1$.

Many published results, concerning the control of the fuzzy system, are based on the PDC principle. The design of the fuzzy controller shares the same antecedent as the fuzzy system and employs a linear state feedback control in the consequent part. For each local dynamics the controller is defined as

Rule $i$ : If $z_{1}(t)$ is $M_{i 1}$ and $z_{2}(t)$ is $M_{i 2} \ldots$ and $z_{p}(t)$ is $M_{i p}$, then

$$
u(t)=-K_{i} x(t), i=1,2, \ldots, r
$$


where $K_{i}$ is the local state feedback gain. Consequently, the defuzzified result is

$$
u(t)=-\sum_{i=1}^{r} \mu_{i}(z) K_{i} x(t)
$$

The system (1) in closed-loop with the fuzzy controller (3) yields the following fuzzy system,

$$
\dot{x}(t)=\sum_{i=1}^{r} \sum_{j=1}^{r} \mu_{i}(z) \mu_{j}(z)\left(A_{i}-B_{i} K_{j}\right) x(t) .
$$

A sufficient condition for the stability is deduced using Lyapunov's direct method. Suppose that a common positive definite matrix $P$ exists, so that the following conditions are satisfied [9].

$$
\left(A_{i}-B_{i} K_{i}\right)^{T} P+P\left(A_{i}-B_{i} K_{i}\right)<0, i=1,2, \ldots, r,
$$

and

$$
\frac{1}{2}\left(A_{i}-B_{i} K_{j}+A_{j}-B_{j} K_{i}\right)^{T} P+\frac{1}{2} P\left(A_{i}-B_{i} K_{j}+A_{j}-B_{j} K_{i}\right)<0,1 \leqslant i<j \leqslant r .
$$

When these conditions are satisfied, the fuzzy system (4) is asymptotically stable. The design work can be transformed into a convex problem [8], which is efficiently solved by linear matrix inequalities optimization. If the solution is feasible, meaning that the stabilization constraints are met, then local state feedback gains are obtained. Relaxed results on stabilization and state feedback $H_{\infty}$ Control conditions for T-S Fuzzy systems were given in [24].

\section{Control of uncertain fuzzy systems}

Motivated by the results of the above section concerning the control of fuzzy-model, we will extend the T-S fuzzy system with the presence of external disturbances [21]. Consider the following T-S fuzzy uncertain model,

Rule $i:$ If $z_{1}(t)$ is $M_{i 1}$ and $z_{2}(t)$ is $M_{i 2} \ldots$ and $z_{p}(t)$ is $M_{i p}$, then

$$
\dot{x}(t)=A_{i} x(t)+B_{i} u(t)+f_{i}(t, x(t)), i=1,2, \ldots, r .
$$

The fuzzy system is then inferred to be

$$
\dot{x}(t)=\sum_{i=1}^{r} \mu_{i}(z)\left(A_{i} x(t)+B_{i} u(t)+f_{i}(t, x(t))\right) .
$$

The function $f_{i}$ represent the uncertain external disturbance of each fuzzy subsystem and are time-varying satisfying the following inequality,

$$
\left\|f_{i}(t, x(t))\right\| \leqslant \alpha_{i}(t)\|x(t)\|+\beta_{i}(t), i=1,2, \ldots, r,
$$

for all $t \geqslant 0$ and $x \in \mathbb{R}^{n}$, where $\alpha_{i}$ and $\beta_{i}$ are known nonnegative continuous functions. 
Remark 2 Inequality (7) means that the time-varying function $f_{i}$ may be bounded and/or unbounded on time. For the knowledge of the authors, this is new.

Suppose the following assumption,

$\left(\mathcal{H}_{1}\right)$ The pairs $\left(A_{i}, B_{i}\right), i=1, \ldots, r$, are controllable, that is each nominal local model is controllable.

The fuzzy control rule is defined as above and we will consider the fuzzy uncertain system (6) Therefore, the closed-loop system with respect the fuzzy control (2 - 3) is given by

$$
\dot{x}(t)=\sum_{i=1}^{r} \sum_{j=1}^{r} \mu_{i}(z) \mu_{j}(z)\left(A_{i}-B_{i} K_{j}\right) x(t)+\sum_{i=1}^{r} \mu_{i}(z) f_{i}(t, x(t)) .
$$

Thus,

$$
\dot{x}(t)=\sum_{i=1}^{r} \mu_{i}^{2} G_{i i} x(t)+2 \sum_{i<j}^{r} \mu_{i} \mu_{j} G_{i j} x(t)+\sum_{i=1}^{r} \mu_{i} f_{i}(t, x(t)),
$$

where

$$
G_{i i}=A_{i}-B_{i} K_{i}
$$

and

$$
G_{i j}=\frac{1}{2}\left(A_{i}-B_{i} K_{j}+A_{j}-B_{j} K_{i}\right) .
$$

The controller synthesis initially considers the stability of the local fuzzy dynamics. That is, the stable feedback gains are determined for every subsystem. Suppose that there exist positive symmetric and definite matrices $P, Q_{i}$, and $Q_{i j}(i<j)$, and some matrices $K_{i}$, $i=1, \ldots, r$, such that the following inequalities [15] hold,

$$
G_{i i}^{T} P+P G_{i i}<-Q_{i}, i=1,2, \ldots, r,
$$

and

$$
G_{i j}^{T} P+P G_{i j}<-Q_{i j}, 1 \leqslant i<j \leqslant r .
$$

Based on this assumption, each nominal local model is controllable and a suitable feedback gain can be obtained.

As a first step, we need to recall what is meant by uniformly ultimately bounded and uniform global practical exponential stability of dynamic systems ( [2], [4], [5]). Consider a system described by

$$
\dot{x}=F(t, x)
$$

with $t \in \mathbb{R}_{+}$is the time and $x \in \mathbb{R}^{n}$ is the state.

Definition 1 The system (11) is said uniformly ultimately bounded if there exists $R>0$, such that for all $R_{1}>0$, there exists a $T=T\left(R_{1}\right)>0$ such that

$$
\left\|x\left(t_{0}\right)\right\| \leqslant R_{1} \Rightarrow\|x(t)\| \leqslant R \text { for all } t \geqslant t_{0}+T \text { and } t_{0} \geqslant 0 .
$$


Definition 2 The system (11) is said uniformly globally practically exponentially stable, if there exists a ball

$$
\mathcal{B}_{\eta}=\left\{x \in \mathbb{R}^{n} /\|x\| \leqslant \eta\right\},
$$

such that $\mathcal{B}_{\eta}$ is uniformly globally practically exponentially stable, it means that, there exists $\eta>0$ such that, for all $\varepsilon>\eta$, there exists $\varepsilon=\varepsilon(\varepsilon)>0$ such that, for all $t_{0} \geqslant 0$, $\left\|x\left(t_{0}\right)\right\| \leqslant \varepsilon$, we have

$$
\|x(t)\| \leqslant \gamma\left\|x\left(t_{0}\right)\right\| e^{-v\left(t-t_{0}\right)}+\eta, \text { for all } t \geqslant t_{0},
$$

with $\gamma>0, v>0$.

The inequality (12) implies that $x(t)$ will be bounded by a small bound $\eta>0$, that is, $\|x(t)\|$ will be small for sufficiently large $t$. It means that (11) will be uniformly ultimately bounded for sufficiently large $t$. If in (12) $\eta$ can be replaced by a smooth map $\eta(t)$ as a function of $t$ which tends to zero as $t$ tends to $+\infty$, then the ultimate bound approaches to zero.

Remark 3 The goal of this paper is to find some conditions on the functions $\alpha_{i}(t)$ and $\beta_{i}(t)$ such that the fuzzy system (8) is globally uniformly practically exponentially stable. If $\beta_{i}(t)=0$, for all $i=1, \ldots, r$, the fuzzy uncertain system (8) has an equilibrium point at the origin. In this case, we can analyze the stability of the closed-loop system behavior for the origin as an equilibrium point. If $\beta_{i}(t) \neq 0$, for some $i=1, \ldots, r$, then the origin can will not be an equilibrium point of the fuzzy uncertain system (8). In this case, we study the convergence of the solutions toward a neighborhood of the origin.

Let

$$
\alpha(t):=\left(\sum_{i=1}^{r} \alpha_{i}(t)^{2}\right)^{\frac{1}{2}},
$$

such that $\alpha$ is bounded and to satisfy: there exists $M_{\alpha}$ a positive scalar constant satisfy,

$$
\int_{0}^{+\infty} \alpha(t) d t \leqslant M_{\alpha}<+\infty .
$$

In the first part, let consider the following assumption,

$\left(\mathcal{H}_{2}\right)$ There exists $M_{\beta}$ a positive scalar constant satisfy,

$$
\int_{0}^{+\infty} \beta^{2}(t) d t \leqslant M_{\beta}<+\infty,
$$

where

$$
\beta(t):=\left(\sum_{i=1}^{r} \beta_{i}(t)^{2}\right)^{\frac{1}{2}}
$$


To find an estimation as in (12), we will impose a restriction on the upper bound of the uncertain term formulated in the following condition,

$\left(\mathcal{H}_{3}\right)$

$$
\alpha(t)<\frac{1}{2} \frac{\lambda_{0}}{\lambda_{\max }(P)}
$$

where $\lambda_{0}=\inf \left\{\left(\lambda_{\min }\left(Q_{i}\right) ; i=1, \ldots, r\right) ;\left(\lambda_{\min }\left(Q_{i j}\right) ; 1 \leqslant i<j \leqslant r\right)\right\}, \lambda_{\min (\max )}$ denotes the smallest (largest) eigenvalue of the matrix.

Remark 4 The inequality (13) is equivalent to the following LMIs,

$$
P<\frac{1}{2 \alpha(t)} Q_{i}, \quad i=1, \ldots, r,
$$

and

$$
P<\frac{1}{2 \alpha(t)} Q_{i j}, \quad 1 \leqslant i<j \leqslant r .
$$

Compared to classical LMI conditions, the new LMIs are a little bit more sever in order to handle the time varying uncertain term.

Remark 5 The matrices $P, Q_{i}, Q_{i j}(i<j)$ and $K_{i}$ can be obtained using the following LMIs,

$$
\begin{aligned}
& X>0 \\
& X<\frac{1}{2 \alpha(t)} X Q_{i} X, \quad i=1, \ldots, r, \\
& X<\frac{1}{2 \alpha(t)} X Q_{i j} X, \quad 1 \leqslant i<j \leqslant r \\
& X A_{i}^{T}+A_{i} X-M_{i}^{T} B_{i}^{T}-B_{i} M_{i}<-X Q_{i} X, \quad i=1, \ldots, r, \\
& X A_{i}^{T}+A_{i} X+X A_{j}^{T}+A_{j} X-M_{j}^{T} B_{i}^{T}-M_{i}^{T} B_{j}^{T}-B_{i} M_{j}-B_{j} M_{i}<-2 X Q_{i j} X, \quad 1 \leqslant i<j \leqslant r, \\
& {\left[\begin{array}{cccc}
Q_{1} & Q_{12} & \ldots & Q_{1 r} \\
Q_{12} & Q_{22} & & \vdots \\
\vdots & & \ddots & Q_{r(r-1)} \\
Q_{1 r} & \ldots & Q_{r(r-1)} & Q_{r}
\end{array}\right]>0}
\end{aligned}
$$

where $X=P^{-1}, K_{i}=M_{i} P$.

Now, one can state the following theorem. 
Theorem 3 Suppose that the assumptions $\left(\mathcal{H}_{1}\right),\left(\mathcal{H}_{2}\right)$ and $\left(\mathcal{H}_{3}\right)$ hold and there exist a common positive definite matrix $P$ and some feedback gain matrices $K_{i}, i=1, \ldots, r$, such that the stability conditions (9-10) are satisfied, then the fuzzy closed-loop system (8) with the control laws (2-3) is guaranteed to be globally uniformly practically exponentially stable.

Proof. Consider the Lyapunov function candidate $V(t, x)=x^{T} P x$. It's derivative with respect to time is given by,

$$
\dot{V}(t, x)=\sum_{i=1}^{r} \mu_{i}^{2} x^{T}\left(G_{i i}^{T} P+P G_{i i}\right) x+2 \sum_{i<j}^{r} \mu_{i} \mu_{j} x^{T}\left(G_{i j}^{T} P+P G_{i j}\right) x+2 x^{T} P \sum_{i=1}^{r} \mu_{i} f_{i}(t, x(t)) .
$$

The first two terms on the right-hand side constitute the derivative of the Lyapunov function $V(x)$ with respect the nominal system, while the third term is the effect of the perturbation. On the one hand, we have

$$
x^{T}\left(G_{i i}^{T} P+P G_{i i}\right) x \leqslant-\lambda_{\min }\left(Q_{i}\right)\|x\|^{2}, i=1,2, \ldots, r,
$$

and

$$
x^{T}\left(G_{i j}^{T} P+P G_{i j}\right) x \leqslant-\lambda_{\min }\left(Q_{i j}\right)\|x\|^{2}, 1 \leqslant i<j \leqslant r .
$$

It follows that,

$$
\dot{V}(t, x) \leqslant-\sum_{i=1}^{r} \mu_{i}^{2} \lambda_{\min }\left(Q_{i}\right)\|x\|^{2}-2 \sum_{i<j}^{r} \mu_{i} \mu_{j} \lambda_{\min }\left(Q_{i j}\right)\|x\|^{2}+2 x^{T} P \sum_{i=1}^{r} \mu_{i} f_{i}(t, x(t)) .
$$

Thus,

$$
\dot{V}(t, x) \leqslant-\left(\sum_{i=1}^{r} \mu_{i}^{2} \lambda_{\min }\left(Q_{i}\right)+2 \sum_{i<j}^{r} \mu_{i} \mu_{j} \lambda_{\min }\left(Q_{i j}\right)\right)\|x\|^{2}+2 x^{T} P \sum_{i=1}^{r} \mu_{i} f_{i}(t, x(t)) .
$$

Then, one gets

$$
\dot{V}(t, x) \leqslant-\lambda_{0}\|x\|^{2} \sum_{i=1}^{r} \sum_{i=1}^{r} \mu_{i} \mu_{j}+2 x^{T} P \sum_{i=1}^{r} \mu_{i} f_{i}(t, x(t)) .
$$

Since,

$$
\sum_{i=1}^{r} \sum_{j=1}^{r} \mu_{i} \mu_{j}=1
$$

then, we have

$$
\dot{V}(t, x) \leqslant-\lambda_{0}\|x\|^{2}+2 x^{T} P \sum_{i=1}^{r} \mu_{i} f_{i}(t, x(t)) .
$$

On the other hand, we have

$$
\left\|\sum_{i=1}^{r} \mu_{i} f_{i}(t, x(t))\right\| \leqslant \sum_{i=1}^{r} \mu_{i}\left(\alpha_{i}(t)\|x\|+\beta_{i}(t)\right) .
$$


Taking into account the above expressions, it follows that

$$
\dot{V}(t, x) \leqslant-\lambda_{0}\|x\|^{2}+2\|x\|\|P\| \sum_{i=1}^{r} \mu_{i}\left(\alpha_{i}(t)\|x\|+\beta_{i}(t)\right) \text {. }
$$

Thus, by using the Cauchy-Schwartz inequality, one has

$$
\dot{V}(t, x) \leqslant-\lambda_{0}\|x\|^{2}+2\|x\|\|P\|\left(\left(\sum_{i=1}^{r} \mu_{i}^{2}\right)^{\frac{1}{2}}\left(\sum_{i=1}^{r} \alpha_{i}(t)^{2}\right)^{\frac{1}{2}}\|x\|+\left(\sum_{i=1}^{r} \mu_{i}^{2}\right)^{\frac{1}{2}}\left(\sum_{i=1}^{r} \beta_{i}(t)^{2}\right)^{\frac{1}{2}}\right) .
$$

It follows that,

$$
\dot{V}(t, x) \leqslant-\lambda_{0}\|x\|^{2}+2\|P\|\left(\sum_{i=1}^{r} \alpha_{i}(t)^{2}\right)^{\frac{1}{2}}\|x\|^{2}+2\|P\|\left(\sum_{i=1}^{r} \beta_{i}(t)^{2}\right)^{\frac{1}{2}}\|x\| .
$$

Hence,

$$
\dot{V}(t, x) \leqslant-\left(\lambda_{0}-2\|P\|\left(\sum_{i=1}^{r} \alpha_{i}(t)^{2}\right)^{\frac{1}{2}}\right)\|x\|^{2}+2\|P\|\left(\sum_{i=1}^{r} \beta_{i}(t)^{2}\right)^{\frac{1}{2}}\|x\| .
$$

Since,

$$
\lambda_{\text {min }}(P)\|x\|^{2} \leqslant V(t, x)=x^{T} P x \leqslant \lambda_{\max }(P)\|x\|^{2},
$$

then, by taking $\|P\|=\lambda_{\max }(P)$, yields

$$
\dot{V}(t, x) \leqslant-\frac{1}{\lambda_{\max }(P)}\left(\lambda_{0}-2 \lambda_{\max }(P) \alpha(t)\right) V(t, x)+2 \frac{\lambda_{\max }(P)}{\lambda_{\text {min }}^{\frac{1}{2}}(P)} \beta(t) V(t, x)^{\frac{1}{2}} .
$$

Let,

$$
\begin{gathered}
a(t)=\frac{1}{\lambda_{\text {max }}(P)}\left(\lambda_{0}-2 \lambda_{\text {max }}(P) \alpha(t)\right), \\
b(t)=2 \frac{\lambda_{\text {max }}(P)}{\lambda_{\text {min }}^{\frac{1}{2}}(P)} \beta(t) .
\end{gathered}
$$

With the previous notations, it follows that

$$
\dot{V}(t, x) \leqslant-a(t) V(t, x)+b(t) V(t, x)^{\frac{1}{2}} .
$$

In the last expression, we make the following change of variable, $w(t)=V(t, x)^{\frac{1}{2}}$. The derivative with respect to time is given by

$$
\dot{w}(t)=\frac{\dot{V}(t, x)}{2 V(t, x)^{\frac{1}{2}}} .
$$


This implies that,

$$
\dot{w}(t) \leqslant-\frac{1}{2} a(t) w(t)+\frac{1}{2} b(t) .
$$

Letting

$$
z(t)=w(t) e^{\frac{1}{2} \int_{t_{0}}^{t} a(s) d s}
$$

it follows that,

$$
\dot{z}(t)=\left(\dot{w}(t)+\frac{1}{2} a(t) w(t)\right) e^{\frac{1}{2} \int_{t_{0}}^{t} a(s) d s} \leqslant \frac{1}{2} b(t) e^{\frac{1}{2} \int_{t_{0}}^{t} a(s) d s} .
$$

Integrating between $t_{0}$ and $t$, one obtains for all $t \geqslant t_{0}$,

$$
z(t) \leqslant z\left(t_{0}\right)+\frac{1}{2} \int_{t_{0}}^{t} b(s) e^{\frac{1}{2} \int_{t_{0}}^{s} a(\xi) d \xi} d s .
$$

By the fact that $z(t)=w(t) e^{\frac{1}{2} \int_{t_{0}}^{t} a(s) d s}$, we obtain

$$
w(t) \leqslant w\left(t_{0}\right) e^{-\frac{1}{2} \int_{t_{0}}^{t} a(s) d s}+\frac{1}{2}\left(\int_{t_{0}}^{t} b(s) e^{\frac{1}{2} \int_{t_{0}}^{s} a(\xi) d \xi} d s\right) e^{-\frac{1}{2} \int_{t_{0}}^{t} a(s) d s} .
$$

Using the forms of $a(t)$ and $b(t)$, we first compute $-\frac{1}{2} \int_{t_{0}}^{t} a(s) d s$.

$$
-\frac{1}{2} \int_{t_{0}}^{t} a(s) d s=-\frac{1}{2} \frac{\lambda_{0}}{\lambda_{\max }(P)}\left(t-t_{0}\right)+\int_{t_{0}}^{t} \alpha(s) d s \leqslant-\frac{1}{2} \frac{\lambda_{0}}{\lambda_{\max }(P)}\left(t-t_{0}\right)+M_{\alpha} .
$$

It follows that, the first term on the right-hand side of (3.12) satisfies,

$$
e^{-\frac{1}{2} \int_{t_{0}}^{t} a(s) d s} \leqslant e^{M_{\alpha}} e^{-\frac{1}{2} \frac{\lambda_{0}}{\lambda_{\max (P)}}\left(t-t_{0}\right)} .
$$

Next, consider the second term on the right-hand side of (3.12). We have,

$$
\begin{aligned}
\frac{1}{2}\left(\int_{t_{0}}^{t} b(s) e^{\frac{1}{2} \int_{t_{0}}^{s} a(\xi) d \xi} d s\right) e^{-\frac{1}{2} \int_{t_{0}}^{t} a(s) d s} \\
=\left(\int_{t_{0}}^{t} \frac{\lambda_{\max }(P)}{\lambda_{\min }^{\frac{1}{2}}(P)} \beta(s) e^{\frac{1}{2} \frac{\lambda_{0}}{\lambda_{\max }(P)}\left(s-t_{0}\right)-\int_{t_{0}}^{s} \alpha(\xi) d \xi} d s\right) e^{M_{\alpha}} e^{-\frac{1}{2} \frac{\lambda_{0}}{\lambda_{\max }(P)}\left(t-t_{0}\right)} .
\end{aligned}
$$


Note that, since $\alpha(t) \geqslant 0$ for all $t \geqslant 0$, then it is clear that

$$
e^{-\int_{t_{0}}^{t} \alpha(\xi) d \xi} \leqslant 1
$$

Thus,

$$
\begin{gathered}
\frac{1}{2}\left(\int_{t_{0}}^{t} b(s) e^{\frac{1}{2} \int_{t_{0}}^{s} a(\xi) d \xi} d s\right) e^{-\frac{1}{2} \int_{t_{0}}^{t} a(s) d s} \leqslant\left(\int_{t_{0}}^{t} \frac{\lambda_{\max }(P)}{\lambda_{\min }^{\frac{1}{2}}(P)} \beta(s) e^{\frac{1}{2} \frac{\lambda_{0}}{\lambda_{\max }(P)}\left(s-t_{0}\right)} d s\right) e^{M_{\alpha}} e^{-\frac{1}{2} \frac{\lambda_{0}}{\lambda_{\max }(P)}\left(t-t_{0}\right)} \\
\leqslant \frac{\lambda_{\max }(P)}{\lambda_{\text {min }}^{\frac{1}{2}}(P)} e^{M_{\alpha}} e^{-\frac{1}{2} \frac{\lambda_{0}}{\lambda_{\max }(P)}\left(t-t_{0}\right)}\left(\int_{t_{0}}^{t}(\beta(s))^{2} d s\right)^{\frac{1}{2}}\left(\int_{t_{0}}^{t} e^{\frac{\lambda_{0}}{\lambda_{\max }(P)}\left(s-t_{0}\right)} d s\right)^{\frac{1}{2}} .
\end{gathered}
$$

Hence,

$$
\frac{1}{2}\left(\int_{t_{0}}^{t} b(s) e^{\frac{1}{2} \int_{t_{0}}^{s} a(\xi) d \xi} d s\right) e^{-\frac{1}{2} \int_{t_{0}}^{t} a(s) d s} \leqslant M_{\beta}^{\frac{1}{2}} e^{M_{\alpha}} \frac{\lambda_{\text {max }}^{\frac{3}{2}}(P)}{\lambda_{\text {min }}^{\frac{1}{2}}(P) \lambda_{0}^{\frac{1}{2}}} .
$$

The inequality (16) in conjunction with (17) and (18), yields

$$
w(t) \leqslant w\left(t_{0}\right) e^{M_{\alpha}} e^{-\frac{1}{2} \frac{\lambda_{0}}{\lambda_{\max }(P)}\left(t-t_{0}\right)}+M_{\beta}^{\frac{1}{2}} e^{M_{\alpha}} \frac{\lambda_{\max }^{\frac{3}{2}}(P)}{\lambda_{\min }^{\frac{1}{2}}(P) \lambda_{0}^{\frac{1}{2}}} .
$$

It follows that,

$$
V(t, x)^{\frac{1}{2}} \leqslant V\left(t_{0}, x\left(t_{0}\right)\right)^{\frac{1}{2}} e^{M_{\alpha}} e^{-\frac{1}{2} \frac{\lambda_{0}}{\lambda_{\max (P)}}\left(t-t_{0}\right)}+M_{\beta}^{\frac{1}{2}} e^{M_{\alpha}} \frac{\lambda_{\text {max }}^{\frac{3}{2}}(P)}{\lambda_{\min }^{\frac{1}{2}}(P) \lambda_{0}^{\frac{1}{2}}} .
$$

Therefore,

$$
\|x(t)\| \leqslant \frac{\lambda_{\text {max }}^{\frac{1}{2}}(P)}{\lambda_{\text {min }}^{\frac{1}{2}}(P)} e^{M_{\alpha}}\left\|x\left(t_{0}\right)\right\| e^{-\frac{1}{2} \frac{\lambda_{0}}{\lambda_{\max }(P)}\left(t-t_{0}\right)}+M_{\beta}^{\frac{1}{2}} e^{M_{\alpha}} \frac{\lambda_{\text {max }}^{\frac{3}{2}}(P)}{\lambda_{\min }(P) \lambda_{0}^{\frac{1}{2}}} .
$$

Hence, we obtain an estimation as in (12) with

$$
\begin{gathered}
\gamma=\frac{\lambda_{\text {max }}^{\frac{1}{2}}(P)}{\lambda_{\text {min }}^{\frac{1}{2}}(P)} e^{M_{\alpha}}, \\
v=\frac{1}{2} \frac{\lambda_{0}}{\lambda_{\max }(P)},
\end{gathered}
$$


and

$$
\eta_{f}=M_{\beta}^{\frac{1}{2}} e^{M_{\alpha}} \frac{\lambda_{\max }^{\frac{3}{2}}(P)}{\lambda_{\min }(P) \lambda_{0}^{\frac{1}{2}}} .
$$

Therefore, $\mathcal{B}_{\eta_{f}}$ is globally uniformly practically exponentially stable.

Remark 6 This bound can be minimized by solving the following optimization problem: Find $P, Q_{i}, Q_{i j} i<j$ and $K_{i}, i, j=1, \ldots, r$, and maximize $\varepsilon_{1}, \varepsilon_{2}, \varepsilon_{3}, \varepsilon_{4}, \varepsilon_{5}$ and $\varepsilon_{6}$ subject to:

$$
\begin{gathered}
P=P^{T}>0, \quad P>\varepsilon_{1} I, \quad-P>-\varepsilon_{2} I, \\
P<\frac{1}{2 \alpha(t)} Q_{i}-\varepsilon_{3} I, \quad i=1, \ldots, r, \\
P<\frac{1}{2 \alpha(t)} Q_{i j}-\varepsilon_{4} I, \quad 1 \leqslant i<j \leqslant r, \\
G_{i i}^{T} P+P G_{i i}<-Q_{i}-\varepsilon_{5} I, i=1, \ldots, r, \\
G_{i j}^{T} P+P G_{i j}<-Q_{i j}-\varepsilon_{6} I, 1 \leqslant i<j \leqslant r,
\end{gathered}
$$

and

$$
\left[\begin{array}{cccc}
Q_{1} & Q_{12} & \ldots & Q_{1 r} \\
Q_{12} & Q_{22} & & \vdots \\
\vdots & & \ddots & Q_{r(r-1)} \\
Q_{1 r} & \ldots & Q_{r(r-1)} & Q_{r}
\end{array}\right]>0
$$

Where $I$ is the matrix identity.

Remark 7 Compared to the existing results, such as the input-output methods and slack matrix method as in ( [10], [11], [25]), in this work the quadratic Lyapunov function and the PDC controller techniques can be used to show the ultimate boundedness of the solutions of the uncertain T-S fuzzy systems, even when the origin is not an equilibrium point of the system. Therefore, we can study the convergence of the solutions toward a neighborhood of the origin and this is what we mean by practical stability.

In the second part, we suppose the following assumption.

$$
\delta(t) \leqslant M_{\delta}, \text { for all } i=1,2, \ldots, r \text { and } t \geqslant 0,
$$

where

$$
\delta(t):=\sum_{i=1}^{r} \mu_{i} \beta_{i}(t)
$$

and $M_{\delta}$ is a positive constant. 
Theorem 4 Suppose that the assumptions $\left(\mathcal{H}_{1}\right),\left(\mathcal{H}^{\prime}{ }_{2}\right)$ and $\left(\mathcal{H}_{3}\right)$ hold and there exist a common positive definite matrix $P$ and some feedback gain matrices $K_{i}, i=1, \ldots, r$, such that the stability conditions $(9-10)$ are satisfied, then the fuzzy closed-loop system (8) with the control laws (2 - 3) is guaranteed to be globally uniformly practically exponentially stable.

Proof. Let consider the Lyapunov function candidate $V(t, x)=x^{T} P x$. It's derivative with respect to time is given by,

$$
\dot{V}(t, x)=\sum_{i=1}^{r} \mu_{i}^{2} x^{T}\left(G_{i i}^{T} P+P G_{i i}\right) x+2 \sum_{i<j}^{r} \mu_{i} \mu_{j} x^{T}\left(G_{i j}^{T} P+P G_{i j}\right) x+2 x^{T} P \sum_{i=1}^{r} \mu_{i} f_{i}(t, x(t)),
$$

then, we have

$$
\dot{V}(t, x) \leqslant-\lambda_{0}\|x\|^{2}+2 x^{T} P \sum_{i=1}^{r} \mu_{i} f_{i}(t, x(t)) .
$$

Thus, by using the following inequality,

$$
\left\|f_{i}(t, x(t))\right\| \leqslant \alpha_{i}(t)\|x\|+\beta_{i}(t),
$$

one has

$$
\dot{V}(t, x) \leqslant-\left(\lambda_{0}-2\|P\|\left(\sum_{i=1}^{r} \alpha_{i}(t)^{2}\right)^{\frac{1}{2}}\right)\|x\|^{2}+2 M_{\delta}\|P\|\|x\| .
$$

Then, by taking $\|P\|=\lambda_{\max }(P)$, yields

$$
\dot{V}(t, x) \leqslant-\frac{1}{\lambda_{\max }(P)}\left(\lambda_{0}-2 \lambda_{\max }(P) \alpha(t)\right) V(t, x)+2 M_{\delta} \frac{\lambda_{\max }(P)}{\lambda_{\text {min }}^{\frac{1}{2}}(P)} V(t, x)^{\frac{1}{2}} .
$$

By using the same idea as in the proof of theorem 1, we obtain the following estimation

$$
\|x(t)\| \leqslant \frac{\lambda_{\max }^{\frac{1}{2}}(P)}{\lambda_{\min }^{\frac{1}{2}}(P)}\left\|x\left(t_{0}\right)\right\| e^{M_{\alpha}} e^{-\frac{1}{2} \frac{\lambda_{0}}{\lambda_{\max }(P)}\left(t-t_{0}\right)}+2 M_{\delta} e^{M_{\alpha}} \frac{\lambda_{\max }^{2}(P)}{\lambda_{\min }(P) \lambda_{0}} .
$$

It follows that,

$$
\mathcal{B}_{\eta}=\left\{x \in \mathbb{R}^{n} /\|x\| \leqslant \eta=2 M_{\delta} e^{M_{\alpha}} \frac{\lambda_{\max }^{2}(P)}{\lambda_{\min }(P) \lambda_{0}}\right\},
$$

is globally uniformly practically exponentially stable.

Motivated by the above results, the design principle can be extended to the T-S fuzzy system with parametric uncertainties. Indeed, one can consider the following T-S fuzzy uncertain model,

Rule $i$ : If $z_{1}(t)$ is $M_{i 1}$ and $z_{2}(t)$ is $M_{i 2} \ldots$ and $z_{p}(t)$ is $M_{i p}$, then

$$
\dot{x}(t)=\left(A_{i}+\Delta A_{i}\right) x(t)+B_{i} u(t)+f_{i}(t, x(t)), i=1,2, \ldots, r .
$$


Notably, the model is almost the same as (5) except for the term $\Delta A_{i}$ which stand for the parametric uncertainties for each subsystem and time-varying with appropriate dimensions. The fuzzy system is then inferred to be

$$
\dot{x}(t)=\sum_{i=1}^{r} \mu_{i}(z)\left(\left(A_{i}+\Delta A_{i}\right) x(t)+B_{i} u(t)+f_{i}(t, x(t))\right) .
$$

Then, let us consider the following assumptions.

$\left(\mathcal{H}_{4}\right)$ The parametric uncertainties $\Delta A_{i}$ is norm bounded and structured, in the form

$$
\Delta A_{i}=\rho_{i}(t) D_{i} E_{i}(t) F_{i},
$$

where $D_{i}$, and $F_{i}$ are known real constant matrices with appropriate dimensions, $E_{i}(t)$, is unknown matrix function which satisfy,

$$
E_{i}^{T}(t) E_{i}(t) \leqslant I, \text { and } E_{i}(t) E_{i}^{T}(t) \leqslant I \text { for all } t \geqslant 0,
$$

and $\rho_{i}(t)$ is a known continuous nonnegative scalar function and $I$ is the identity matrix of appropriate dimension. Let

$$
\rho(t):=\left(\sum_{i=1}^{r} \rho_{i}^{2}(t)\right)^{\frac{1}{2}}
$$

such that, there exists $M_{\rho}$ a positive scalar constant satisfy,

$$
\int_{0}^{+\infty} \rho(t) d t \leqslant M_{\rho}<+\infty .
$$

$\left(\mathcal{H}_{5}\right)$ We suppose that $\rho(t)$ satisfies the following restriction,

$$
\left(\lambda_{0}-2 \sigma_{1}\left(\lambda_{\max }^{2}(P)+\sigma_{2}\right) \rho(t)-2 \lambda_{\max }(P) \alpha(t)\right)>0, \text { for all } t \geqslant 0,
$$

where $\lambda_{0}=\inf \left\{\left(\lambda_{\min }(Q i) ; i=1, \ldots, r\right),\left(\lambda_{\min }(Q i j) ; 1<i \leqslant j<r\right)\right\}, \sigma_{1}=\max \left(\left\|D_{i}\right\|^{2}, i=\right.$ $1, \ldots, r)$ and $\sigma_{2}=\max \left(\left\|F_{i}\right\|^{2}, i=1, \ldots, r\right)$.

Remark 8 The inequality (22) is equivalent to the following LMIs,

$$
\begin{gathered}
P<\frac{1}{2 \sigma \rho(t)} Q_{i}, \quad i=1, \ldots, r \\
P<\frac{1}{2 \alpha(t)} Q_{i}, \quad i=1, \ldots, r
\end{gathered}
$$




$$
P<\frac{1}{2 \sigma \rho(t)} Q_{i j}, \quad 1 \leqslant i<j \leqslant r,
$$

and

$$
P<\frac{1}{2 \alpha(t)} Q_{i j}, \quad 1 \leqslant i<j \leqslant r
$$

where $\sigma=\inf \left(\sigma_{1}, \sigma_{2}\right)$.

Remark 9 Similar to the remark (8), in this case the matrices $P, Q_{i}, Q_{i j}(i<j)$ and $K_{i}$ can be obtained using the following LMIs,

$$
\begin{aligned}
& X>0, \\
& X<\frac{1}{2 \alpha(t)} X Q_{i} X, \quad i=1, \ldots, r, \\
& X<\frac{1}{2 \sigma \rho(t)} X Q_{i} X, \quad i=1, \ldots, r, \\
& X<\frac{1}{2 \alpha(t)} X Q_{i j} X, \quad 1 \leqslant i<j \leqslant r, \\
& X<\frac{1}{2 \sigma \rho(t)} X Q_{i j} X, \quad 1 \leqslant i<j \leqslant r, \\
& X A_{i}^{T}+A_{i} X-M_{i}^{T} B_{i}^{T}-B_{i} M_{i}<-X Q_{i} X, \quad i=1, \ldots, r, \\
& X A_{i}^{T}+A_{i} X+X A_{j}^{T}+A_{j} X-M_{j}^{T} B_{i}^{T}-M_{i}^{T} B_{j}^{T}-B_{i} M_{j}-B_{j} M_{i}<-2 X Q_{i j} X, \quad 1 \leqslant i<j \leqslant r, \\
& {\left[\begin{array}{cccc}
Q_{1} & Q_{12} & \ldots & Q_{1 r} \\
Q_{12} & Q_{22} & & \vdots \\
\vdots & & \ddots & Q_{r(r-1)} \\
Q_{1 r} & \ldots & Q_{r(r-1)} & Q_{r}
\end{array}\right]>0,}
\end{aligned}
$$

where $X=P^{-1}, K_{i}=M_{i} P$.

Then, let consider the following theorem.

Theorem 5 Suppose that the assumptions $\left(\mathcal{H}_{1}\right),\left(\mathcal{H}_{2}\right),\left(\mathcal{H}_{4}\right)$ and $\left(\mathcal{H}_{5}\right)$ hold and there exist a common positive definite matrix $P$ and some feedback gain matrices $K_{i}, i=1, \ldots, r$, such that the stability conditions (9-10) are satisfied, then the fuzzy closed-loop system (21) with the control laws (2-3) is guaranteed to be globally uniformly practically exponentially stable. 
Proof. Consider the Lyapunov function candidate $V(t, x)=x^{T} P x$. It's derivative with respect to time is given by,

$$
\begin{aligned}
\dot{V}(t, x) & =\sum_{i=1}^{r} \mu_{i}^{2} x^{T}\left(G_{i i}^{T} P+P G_{i i}\right) x+2 \sum_{i<j}^{r} \mu_{i} \mu_{j} x^{T}\left(G_{i j}^{T} P+P G_{i j}\right) x+2 x^{T} \sum_{i=1}^{r} \mu_{i} P \Delta A_{i} x \\
& +2 x^{T} P \sum_{i=1}^{r} \mu_{i} f_{i}(t, x(t)) .
\end{aligned}
$$

By the fact that,

$$
\begin{aligned}
2 x^{T} P \Delta A_{i} x & =x^{T}\left(\Delta A_{i}^{T} P+P \Delta A_{i}\right) x \\
2 x^{T} P \Delta A_{i} x & \leqslant \rho_{i}(t) x^{T} P D_{i} D_{i}^{T} P x+\rho_{i}(t) F_{i}^{T} F_{i} x \\
& \leqslant \rho_{i}(t) x^{T}\left(P D_{i} D_{i}^{T} P+F_{i}^{T} F_{i}\right) x .
\end{aligned}
$$

we have

$$
\begin{aligned}
\dot{V}(t, x) & \leqslant-\lambda_{0}\|x\|^{2}+2 \sum_{i=1}^{r} \mu_{i} \rho_{i}(t)\|P\|^{2}\left\|D_{i}\right\|^{2}\|x\|^{2}+2 \sum_{i=1}^{r} \mu_{i} \rho_{i}(t)\left\|F_{i}\right\|^{2}\|x\|^{2} \\
& +2 \lambda_{\max }(P) \alpha(t)\|x\|^{2}+2 \lambda_{\max }(P) \beta(t)\|x\| .
\end{aligned}
$$

Then,

$$
\begin{aligned}
\dot{V}(t, x) & \leqslant-\lambda_{0}\|x\|^{2}+2 \sigma_{1} \lambda_{\max }^{2}(P) \sum_{i=1}^{r} \mu_{i} \rho_{i}(t)\|x\|^{2}+2 \sigma_{2} \sum_{i=1}^{r} \mu_{i} \rho_{i}(t)\|x\|^{2} \\
& +2 \lambda_{\max }(P) \alpha(t)\|x\|^{2}+2 \lambda_{\max }(P) \beta(t)\|x\| .
\end{aligned}
$$

By using the Cauchy-Schwartz inequality, one has

$$
\begin{aligned}
\dot{V}(t, x) & \leqslant-\lambda_{0}\|x\|^{2}+2 \sigma_{1} \lambda_{\text {max }}^{2}(P) \rho(t)\|x\|^{2}+2 \sigma_{2} \rho(t)\|x\|^{2}+2 \lambda_{\max }(P) \alpha(t)\|x\|^{2} \\
& +2 \lambda_{\max }(P) \beta(t)\|x\| .
\end{aligned}
$$

It follows that,

$$
\begin{aligned}
\dot{V}(t, x) & \leqslant-\left(\lambda_{0}-2\left(\sigma_{1} \lambda_{\max }^{2}(P)+\sigma_{2}\right) \rho(t)-2 \lambda_{\max }(P) \alpha(t)\right)\|x\|^{2} \\
& +2 \lambda_{\max }(P) \beta(t)\|x\| .
\end{aligned}
$$

Then,

$$
\begin{gathered}
\dot{V}(t, x) \leqslant-\frac{1}{\lambda_{\max }(P)}\left(\lambda_{0}-2\left(\sigma_{1} \lambda_{\max }^{2}(P)+\sigma_{2}\right) \rho(t)-2 \lambda_{\max }(P) \alpha(t)\right) V(t, x) \\
+2 \frac{\lambda_{\max }(P)}{\lambda_{\min }^{\frac{1}{2}}(P)} \beta(t) V(t, x)^{\frac{1}{2}} .
\end{gathered}
$$


Let,

$$
\begin{gathered}
a(t)=\frac{1}{\lambda_{\max }(P)}\left(\lambda_{0}-2\left(\sigma_{1} \lambda_{\max }^{2}(P)+\sigma_{2}\right) \rho(t)-2 \lambda_{\max }(P) \alpha(t)\right), \\
b(t)=2 \frac{\lambda_{\max }(P)}{\lambda_{\text {min }}^{\frac{1}{2}}(P)} \beta(t) .
\end{gathered}
$$

It follows that

$$
\dot{V}(t, x) \leqslant-a(t) V(t, x)+b(t) V(t, x)^{\frac{1}{2}} .
$$

Using the same idea as in the proofs of theorems 1 and 2 we obtain the following estimation of the state,

$$
\|x(t)\| \leqslant \frac{\lambda_{\text {max }}^{\frac{1}{2}}(P)}{\lambda_{\text {min }}^{\frac{1}{2}}(P)} e^{\rho M_{\rho}} e^{M_{\alpha}}\left\|x\left(t_{0}\right)\right\| e^{-\frac{1}{2} \frac{\lambda_{0}}{\lambda_{\max }(P)}\left(t-t_{0}\right)}+M_{\beta}^{\frac{1}{2}} e^{\rho M_{\rho}} e^{M_{\alpha}} \frac{\lambda_{\text {max }}^{\frac{3}{2}}(P)}{\lambda_{\min }(P) \lambda_{0}^{\frac{1}{2}}}
$$

where

$$
\rho=\frac{\sigma_{1} \lambda_{\max }^{2}(P)+\sigma_{2}}{\lambda_{\max }(P)}
$$

Here, we obtain an estimation as in (12) with

$$
\begin{gathered}
\gamma=\frac{\lambda_{\text {max }}^{\frac{1}{2}}(P)}{\lambda_{\text {min }}^{\frac{1}{2}}(P)} e^{\rho M_{\rho}} e^{M_{\alpha}} \\
v=\frac{1}{2} \frac{\lambda_{0}}{\lambda_{\max }(P)}
\end{gathered}
$$

and

$$
\eta_{\rho \alpha}=M_{\beta}^{\frac{1}{2}} e^{\rho M_{\rho}} e^{M_{\alpha}} \frac{\lambda_{\max }^{\frac{3}{2}}(P)}{\lambda_{\min }(P) \lambda_{0}^{\frac{1}{2}}}
$$

So, $\mathcal{B}_{\eta_{\rho \alpha}}$ is uniformly globally practically exponentially stable.

Corollary 1 If we suppose that the assumptions $\left(\mathcal{H}_{1}\right),\left(\mathcal{H}^{\prime}{ }_{2}\right),\left(\mathcal{H}_{4}\right)$ and $\left(\mathcal{H}_{5}\right)$ hold and there exist a common positive definite matrix $P$ and some feedback gain matrices $K_{i}, i=$ $1, \ldots, r$, such that the stability conditions (9-10) are satisfied, then the fuzzy closed-loop system (21) with the control laws (2-3) is guaranteed to be uniformly globally practically exponentially stable such that the ball,

$$
\mathcal{B}_{\eta_{\rho \alpha}}=\left\{x \in \mathbb{R}^{n} /\|x\| \leqslant \eta=2 M_{\delta} e^{\rho M_{\rho}} e^{M_{\alpha}} \frac{\lambda_{\max }^{2}(P)}{\lambda_{\min }(P) \lambda_{0}}\right\}
$$

is globally uniformly practically exponentially stable. 
According to the above analysis, the design procedure for uncertain Takagi-Sugeno fuzzy systems is summarized as follows.

Step 1: Confirm that assumption $\left(\mathcal{H}_{1}\right)$ is satisfied for the designed system.

Step 2: Verify that the functions $\alpha(t), \rho(t)$ and $\beta(t)$ satisfy the assumptions of integrability.

Step 3: Solve the LMI problem indicated in remark 3.8 and obtain $P, Q_{i}, Q_{i j}(i<j)$, and $K_{i}, i=1, \ldots, r$.

Step 4: Simulate the system in order to plot its trajectories.

\section{Simulation examples}

To illustrate the proposed fuzzy control approach we propose the following examples.

Example 1 Consider a flexible-joint robot arm. The system is described by the following equations ( [28]):

$$
\begin{gathered}
I_{1} \ddot{\theta}_{1}(t)+m g l \sin \left(\theta_{1}\right)+k\left(\theta_{1}-\theta_{2}\right)=0, \\
I_{2} \ddot{\theta}_{2}(t)+k\left(\theta_{2}-\theta_{1}\right)=u .
\end{gathered}
$$

where $u$ is the torque input, $I_{1}$ is the link inertia, $I_{2}$ is the motor inertia, $m$ is the mass, $g$ is the gravity constant, $l$ is the link length, $k$ is the stiffness, $\theta_{1}$ and $\theta_{2}$ are the angular positions of the first and second joints respectively. Let $x_{1}=\theta_{1}, x_{2}=\dot{\theta}_{1}, x_{3}(t)=\theta_{2}$, $x_{4}=\dot{\theta}_{2}$. The dynamic equations (27) and (28) can be rewritten as

$$
\left\{\begin{array}{l}
\dot{x}_{1}(t)=x_{2}(t) \\
\dot{x}_{2}(t)=I_{1}^{-1}\left(-m g l \sin \left(x_{1}(t)\right)+k x_{3}(t)-k x_{1}(t)\right) \\
\dot{x}_{3}(t)=x_{4}(t) \\
\dot{x}_{4}(t)=I_{2}^{-1}\left(k\left(x_{1}-x_{3}\right)+u(t)\right),
\end{array}\right.
$$

where $x(t)=\left[\begin{array}{llll}x_{1}(t) & x_{2}(t) & x_{3}(t) & x_{4}(t)\end{array}\right]^{T}$, is the state vector. One can represent exactly the system by the following two-rule fuzzy model:

Rule $1:$ If $x_{1}$ is $M_{11}$ then

$$
\dot{x}(t)=A_{1} x(t)+B_{1} u(t)
$$

Rule 2: If $x_{1}$ is $M_{21}$ then

$$
\dot{x}(t)=A_{2} x(t)+B_{2} u(t),
$$


where

$$
\begin{gathered}
A_{1}=\left[\begin{array}{cccc}
0 & 1 & 0 & 0 \\
(m g l-k) I_{1}^{-1} & 0 & k I_{1}^{-1} & 0 \\
0 & 0 & 0 & 1 \\
k I_{2}^{-1} & 0 & -k I_{2}^{-1} & 0
\end{array}\right], B_{1}=\left[\begin{array}{c}
0 \\
0 \\
0 \\
I_{2}^{-1}
\end{array}\right], \\
A_{2}=\left[\begin{array}{cccc}
(-m g l-k) I_{1}^{-1} & 0 & k I_{1}^{-1} & 0 \\
0 & 0 & 0 & 1 \\
k I_{2}^{-1} & 0 & -k I_{2}^{-1} & 0
\end{array}\right], B_{2}=\left[\begin{array}{c}
0 \\
0 \\
0 \\
I_{2}^{-1}
\end{array}\right] .
\end{gathered}
$$

The membership functions for rule 1 and 2 are respectively:

$$
\mu_{1}\left(x_{1}\right)=\left\{\begin{array}{ll}
\frac{1}{2}-\frac{\sin \left(x_{1}\right)}{2 x_{1}} & \text { if } x_{1} \neq 0 \\
0 & \text { if } x_{1}=0
\end{array} \text { and } \mu_{2}\left(x_{1}\right)=1-\mu_{1}\left(x_{1}\right)\right.
$$

In this simulation, we choose $I_{1}=I_{2}=1 \mathrm{kgm}^{2}, m=0.01 \mathrm{~kg}, k=0.05 \mathrm{Nm} / \mathrm{rad}, l=1 \mathrm{~m}$, $g=9.8 m s^{-2}$. Using an LMI optimisation algorithm, we obtain:

$$
P=\left[\begin{array}{llll}
0.0439 & 0.1845 & 0.0155 & 0.0081 \\
0.1845 & 0.8899 & 0.0754 & 0.0424 \\
0.0155 & 0.0754 & 0.0067 & 0.0037 \\
0.0081 & 0.0424 & 0.0037 & 0.0026
\end{array}\right]
$$

the following feedback gains:

$$
K_{1}=\left[\begin{array}{llll}
26.2016 & 131.2200 & 11.7453 & 6.7109
\end{array}\right]
$$

and

$$
K_{2}=\left[\begin{array}{llll}
17.7564 & 90.4835 & 8.2953 & 4.7677
\end{array}\right] .
$$

and the following positive definite matrices:

$$
Q_{1}=\left[\begin{array}{cccc}
46.8501 & -23.1530 & 11.5634 & -0.2938 \\
-23.1530 & 13.6945 & -12.3291 & 0.1252 \\
11.5634 & -12.3291 & 228.6668 & 0.8527 \\
-0.2938 & 0.1252 & 0.8527 & 292.7310
\end{array}\right]
$$




$$
Q_{2}=\left[\begin{array}{cccc}
46.8501 & -2.8481 & 11.5634 & -0.2282 \\
-2.8481 & 2.4814 & -8.6590 & -0.0128 \\
11.5634 & -8.6590 & 228.6668 & 0.8683 \\
-0.2282 & -0.0128 & 0.8683 & 292.7303
\end{array}\right]
$$

and

$$
Q_{12}=\left[\begin{array}{cccc}
416.0678 & 21.4119 & -4.9959 & -2.3294 \\
21.4119 & 482.9281 & 9.8489 & 1.4868 \\
-4.9959 & 9.8489 & 297.6419 & 1.3142 \\
-2.3294 & 1.4868 & 1.3142 & 292.7490
\end{array}\right] \text {. }
$$

It can be easily shown that the following stability conditions are satisfied:

$$
G_{i i}^{T} P+P G_{i i}<-Q_{i}, i=1,2,
$$

and

$$
G_{12}^{T} P+P G_{12}<-Q_{12}
$$

Then, we have

$$
\lambda_{\min }(P)=0.0003, \lambda_{\max }(P)=\|P\|=0.9367
$$

and

$$
\lambda_{0}=\inf \left\{\left(\lambda_{\min }\left(Q_{i}\right) ; i=1,2\right),\left(\lambda_{\min }\left(Q_{12}\right)\right)\right\}=1.6513 .
$$

The resulting PDC control law is as follows:

Rule 1: If $x_{1}$ is $M_{11}$ then

$$
u(t)=-K_{1} x(t)
$$

Rule 2: If $x_{1}$ is $M_{21}$ then

$$
u(t)=-K_{2} x(t) .
$$

That is,

$$
u(t)=-\mu_{1}\left(x_{1}(t)\right) K_{1} x(t)-\mu_{2}\left(x_{1}(t)\right) K_{2} x(t) .
$$

This nonlinear control law guarantees the stability of the fuzzy control system (fuzzy model + PDC control). Fig. 1 shows the response of the system using fuzzy model with the PDC control for initial condition $x_{1}=1, x_{2}=0, x_{3}=0$ and $x_{4}=0$.

Now, we introduce the external disturbances and we approximate the system by the following two-rule fuzzy model:

Rule 1 : If $x_{1}$ is $M_{11}$ then

$$
\dot{x}(t)=A_{1} x(t)+B_{1} u(t)+f_{1}(t, x(t))
$$

Rule 2 : If $x_{1}$ is $M_{21}$ then

$$
\dot{x}(t)=A_{2} x(t)+B_{2} u(t)+f_{2}(t, x(t)),
$$



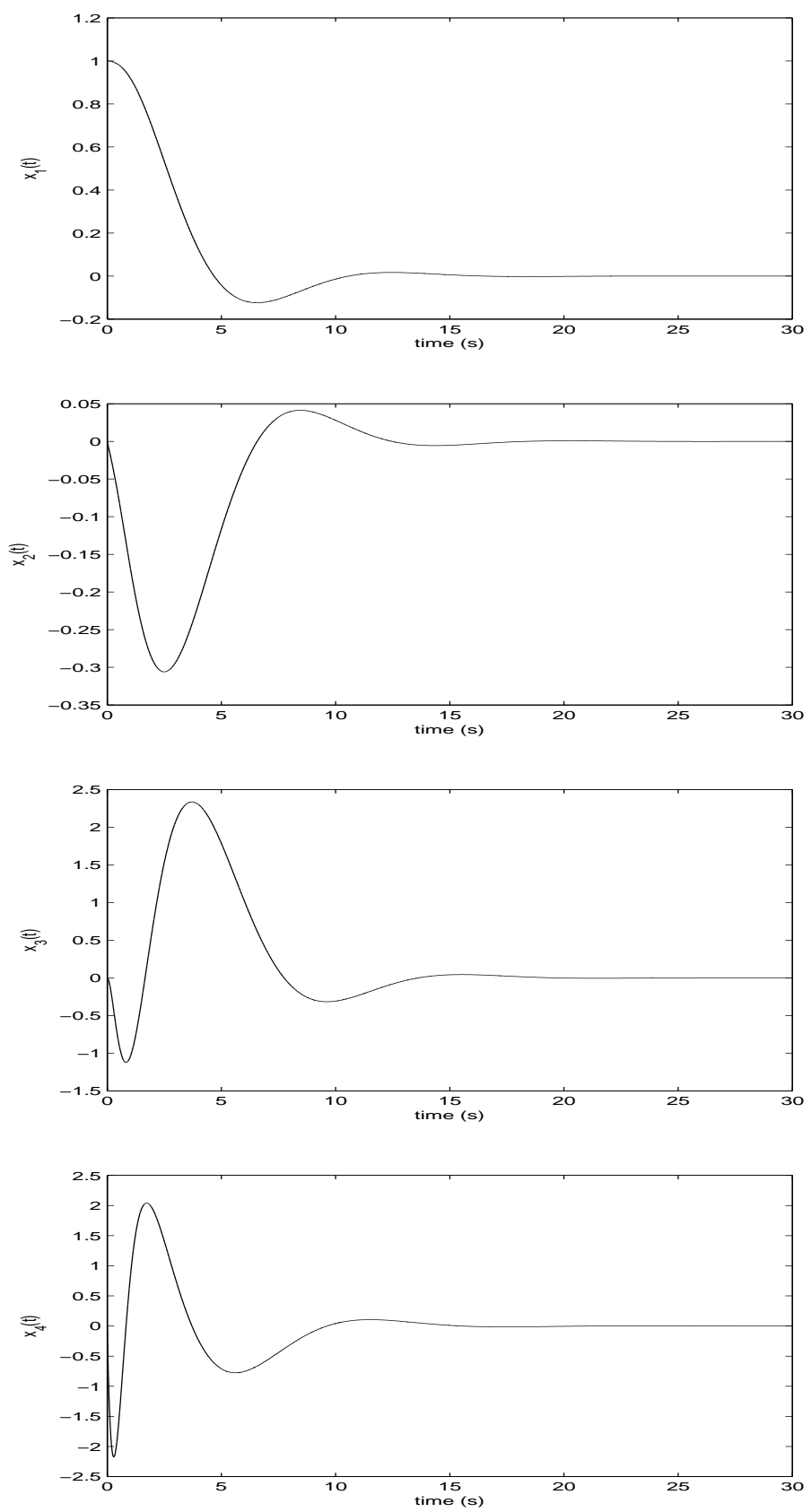

Figure 1: The state of the controlled system 
where

$$
f_{1}(t, x(t))=f_{2}(t, x(t))=\left[\begin{array}{c}
0 \\
0 \\
\frac{\lambda_{0}}{4 \lambda_{\max }(P)\left(1+t^{2}\right)} x_{2}+\frac{1}{2-\sin (t)^{2}} \\
0
\end{array}\right] .
$$

We can see that

$$
\left\|f_{1}(t, x)\right\|=\left\|f_{2}(t, x)\right\| \leqslant \frac{\lambda_{0}}{4 \lambda_{\max }(P)\left(1+t^{2}\right)}\|x\|+\frac{1}{2-\sin (t)^{2}}, \text { for all } t \geqslant 0 .
$$

Therefore, we can choose

$$
\alpha_{1}(t)=\alpha_{2}(t)=\frac{\lambda_{0}}{4 \lambda_{\max }(P)\left(1+t^{2}\right)},
$$

and

$$
\beta_{1}(t)=\beta_{2}(t)=\frac{1}{2-\sin (t)^{2}}
$$

It follows that,

$$
\alpha(t)=(2)^{\frac{1}{2}} \alpha_{1}(t), \text { and } \delta(t)=\beta_{1}(t)
$$

Since

$$
\int_{0}^{+\infty} \alpha(s) d s=\frac{\lambda_{0} \pi}{2 \lambda_{\max }(P)}, \text { and } \delta(t) \leqslant 1,
$$

then we can choose $M_{\alpha}=\frac{\lambda_{0} \pi}{2 \lambda_{\max }(P)}$ and $M_{\delta}=1$. Thus, by using theorem 1 the trajectories of the system are globally uniformly exponentially convergent to the following ball,

$$
\mathcal{B}_{\eta}=\left\{x \in \mathbb{R}^{2} /\|x\| \leqslant \eta=2 M_{\delta} e^{M_{\alpha}} \frac{\lambda_{\max }^{2}(P)}{\lambda_{\min }(P) \lambda_{0}}=56479\right\} .
$$

Fig. 2 shows the response of the flexible-joint robot arm system for initial condition $x_{1}=1, x_{2}=0, x_{3}=0$ and $x_{4}=0$. Also, it shows that the trajectories of the system are globally uniformly ultimately bounded and they converge toward a neighborhood of the origin, under external disturbances. 

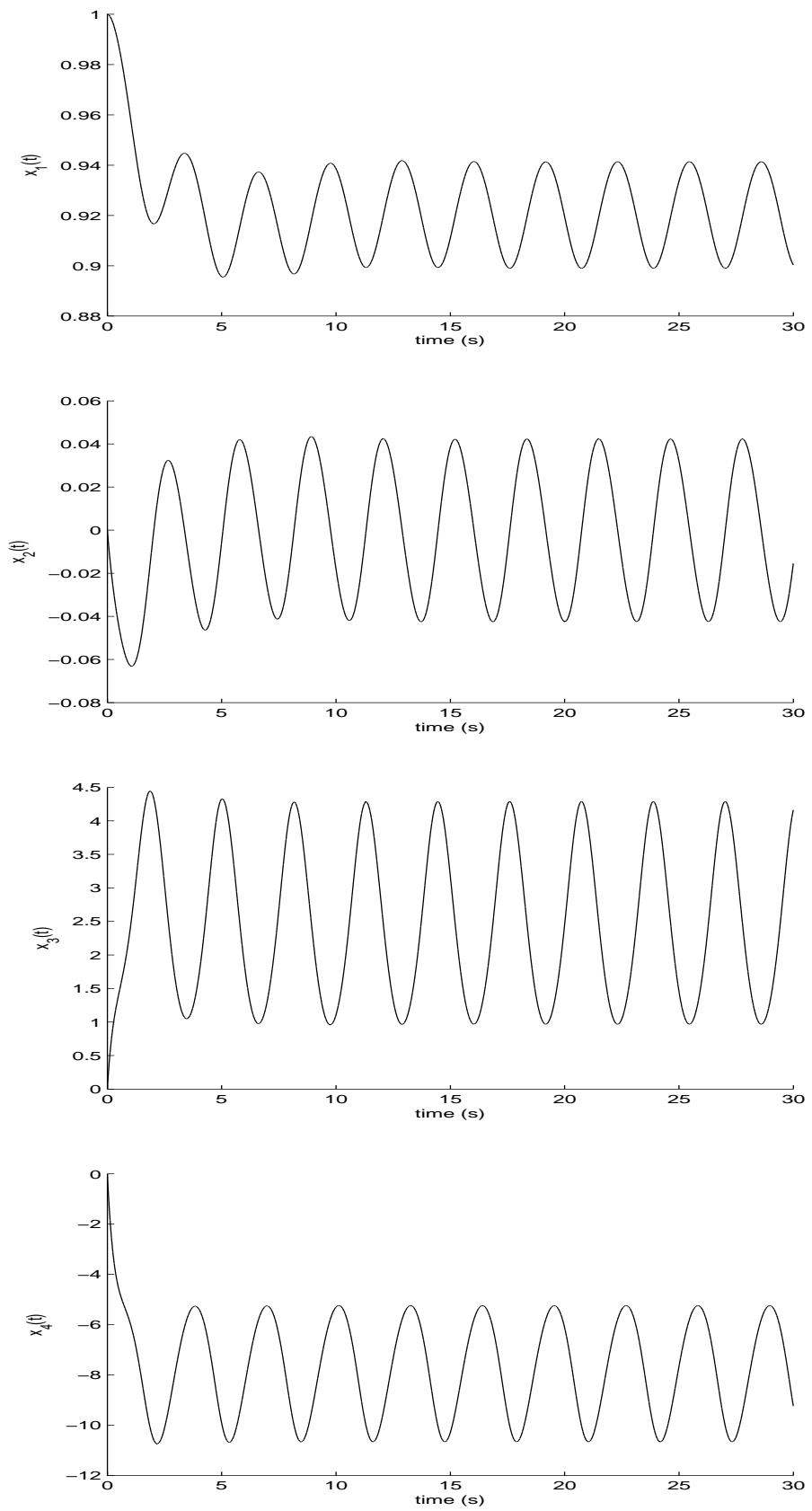

Figure 2: The state of the controlled system under external disturbances 
Example 2 Consider the following nonlinear fuzzy planar system,

$$
\begin{aligned}
& \dot{x}_{1}=-2 x_{1}+\sin \left(x_{1}\right) u \\
& \dot{x}_{2}=x_{1} \sin \left(x_{1}\right)+u,
\end{aligned}
$$

where $x(t)=\left[\begin{array}{ll}x_{1}(t) & x_{2}(t)\end{array}\right]^{T} \in \mathbb{R}^{2}$ is the state vector and $u(t)$ is the input vector.

One can represent exactly the system by the following two-rule fuzzy model:

Rule 1 : If $x_{1}$ is $M_{11}$ then

$$
\dot{x}(t)=A_{1} x(t)+B_{1} u(t)
$$

Rule 2 : If $x_{1}$ is $M_{21}$ then

$$
\dot{x}(t)=A_{2} x(t)+B_{2} u(t)
$$

where

$$
\begin{gathered}
A_{1}=\left[\begin{array}{ll}
-2 & 0 \\
-1 & 0
\end{array}\right], \quad B_{1}=\left[\begin{array}{c}
-1 \\
1
\end{array}\right], \\
A_{2}=\left[\begin{array}{ll}
-2 & 0 \\
1 & 0
\end{array}\right], \quad B_{2}=\left[\begin{array}{l}
1 \\
1
\end{array}\right],
\end{gathered}
$$

We define the membership functions as

$$
\mu_{1}\left(x_{1}(t)\right)=\frac{1-\sin \left(x_{1}(t)\right)}{2} \text { and } \mu_{2}\left(x_{1}(t)\right)=\frac{\sin \left(x_{1}(t)\right)+1}{2} .
$$

Using an LMI optimisation algorithm, yields

$$
P=\left[\begin{array}{ll}
0.0377 & 0.0000 \\
0.0000 & 0.0183
\end{array}\right]
$$

the following feedback gains:

$$
K_{1}=\left[\begin{array}{ll}
-0.0452 & 0.7962
\end{array}\right] \text { and } K_{2}=\left[\begin{array}{ll}
0.0452 & 0.7962
\end{array}\right],
$$

and the matrices:

$Q_{1}=\left[\begin{array}{cc}0.0771 & -0.0063 \\ -0.0063 & 0.0145\end{array}\right], Q_{2}=\left[\begin{array}{ll}0.0771 & 0.0063 \\ 0.0063 & 0.0145\end{array}\right]$ and $Q_{12}=\left[\begin{array}{cc}0.1024 & 0.0000 \\ 0.0000 & 0.0196\end{array}\right]$.

Then, we have

$$
\lambda_{\text {min }}(P)=0.0183, \lambda_{\max }(P)=\|P\|=0.0377
$$

and

$$
\lambda_{0}=\inf \left\{\left(\lambda_{\min }\left(Q_{i}\right) ; i=1,2\right),\left(\lambda_{\min }\left(Q_{12}\right)\right)\right\}=0.0139
$$




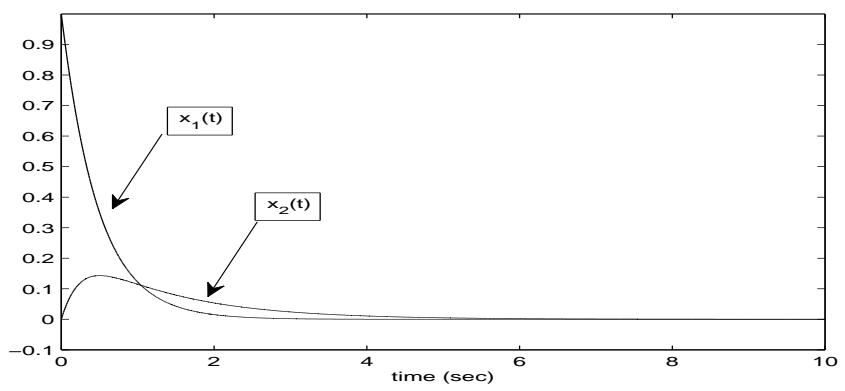

Figure 3: The state responses of the system

Fig. 3 shows the stability of the fuzzy control system (4.1) and (4.2) (fuzzy model + PDC control) with $x_{1}=1$ and $x_{2}=0$ as initial condition.

Now, we introduce parametric uncertainties and external disturbances and we approximate the system by the following two-rule fuzzy models:

Rule 1 : If $x_{1}$ is $M_{11}$ then

$$
\dot{x}(t)=\left(A_{1}+\Delta A_{1}\right) x(t)+B_{1} u(t)+f_{1}(t, x(t))
$$

Rule 2 : If $x_{1}$ is $M_{21}$ then

$$
\dot{x}(t)=\left(A_{2}+\Delta A_{2}\right) x(t)+B_{2} u(t)+f_{2}(t, x(t))
$$

where

$$
\begin{aligned}
\Delta A_{1} & =\rho_{1}(t) F_{1}^{T} E_{1}(t) F_{1}, \\
\Delta A_{2} & =\rho_{2}(t) F_{2}^{T} E_{2}(t) F_{2},
\end{aligned}
$$

with

$$
F_{1}=F_{2}=\left[\begin{array}{ll}
0.1 & 0.1
\end{array}\right] \text { and } \rho_{1}(t)=\rho_{2}(t)=\frac{\lambda_{0}}{4(2)^{\frac{1}{2}}\left(\|F\|^{2} \lambda_{\text {max }}^{2}(P)+\|F\|^{2}\right)\left(1+t^{2}\right)},
$$

and

$$
f_{1}(t, x(t))=f_{2}(t, x(t))=\left[\begin{array}{c}
\mu_{1}+\mu_{2} \\
0
\end{array}\right]
$$

On the one hand, we can see that

$$
\rho(t)=\left(\sum_{i=1}^{r} \rho_{i}(t)^{2}\right)^{\frac{1}{2}}=\frac{\lambda_{0}}{4\left(\left\|F_{1}\right\|^{2} \lambda_{\text {max }}^{2}(P)+\left\|F_{1}\right\|^{2}\right)\left(1+t^{2}\right)},
$$


also

$$
\begin{aligned}
\int_{0}^{+\infty} \rho(s) d s & =\frac{\lambda_{0}}{4\left(\left\|F_{1}\right\|^{2} \lambda_{\text {max }}^{2}(P)+\left\|F_{1}\right\|^{2}\right)} \int_{0}^{+\infty} \frac{1}{\left(1+s^{2}\right)} d s \\
& =\frac{\pi \lambda_{0}}{8\left(\left\|F_{1}\right\|^{2} \lambda_{\text {max }}^{2}(P)+\left\|F_{1}\right\|^{2}\right)},
\end{aligned}
$$

therefore, we can get

$$
M_{\rho}=\frac{\pi \lambda_{0}}{8\left(\left\|F_{1}\right\|^{2} \lambda_{\max }^{2}(P)+\left\|F_{1}\right\|^{2}\right)} .
$$

On the other hand, we have

$$
\left\|f_{1}(t, x(t))\right\|=\left\|f_{2}(t, x(t))\right\| \leqslant 1,
$$

then we can get

$$
\alpha_{1}(t)=\alpha_{2}(t)=0, \quad \beta_{1}(t)=\beta_{2}(t)=1 \quad \text { and } \delta(t)=\sum_{i=1}^{2} \mu_{i} \beta_{i}(t)=1
$$

therefore, we can choose $M_{\alpha}=0$ and $M_{\delta}=1$. Thus, by using Corollary (3.4), it follows that, the system is uniformly globally exponentially converge to the following ball,

$$
\mathcal{B}_{\eta_{\rho \alpha}}=\left\{x \in \mathbb{R}^{2} /\|x\| \leqslant \eta=2 M_{\delta} e^{\rho M_{\rho}} e^{M_{\alpha}} \frac{\lambda_{\text {max }}^{2}(P)}{\lambda_{\min }(P) \lambda_{0}}=11.2365\right\} .
$$

where $\rho=\left\|F_{1}\right\|^{2} \lambda_{\text {max }}^{2}(P)+\left\|F_{1}\right\|^{2}$. The simulation results with initial conditions $x_{1}=1$ and $x_{2}=0$ are shown in figure 4 . It shows that the trajectories of the system converge toward a neighborhood of the origin, under parametric uncertainties and external disturbances.

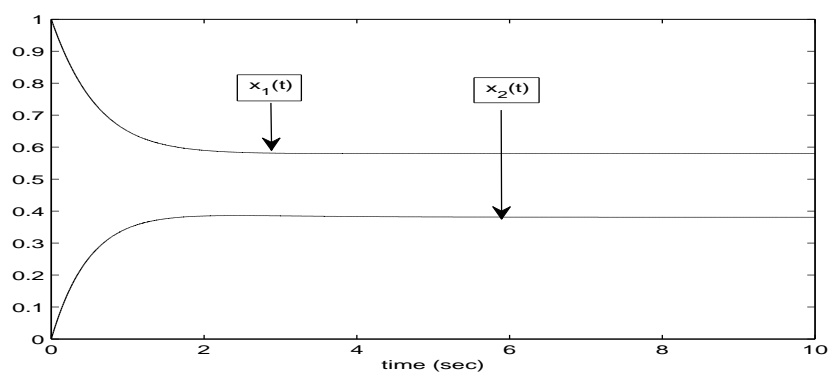

Figure 4: The state responses of the system under parametric uncertainties and external disturbances 


\section{Conclusion}

In this paper, we have studied the global uniform practical exponential stability for a class of uncertain T-S fuzzy systems in term of convergence toward a neighborhood of the origin. The uncertainties are supposed uniformly to be bounded by known integrable functions. We have used quadratic Lyapunov function and parallel distributed compensation (PDC) controller techniques to show the global uniform practical exponential stability of the closed-loop system. Therefore, new LMIs are obtained for the controller in order to handel the uncertainties. Then, systems' performance is proved by adjusting the practical stability conditions. The effectiveness of the proposed theory is illustrated by computer simulation of a flexible-joint robot arm and a planar systems.

\section{References}

[1] A. Benzaouia and A. El Hajuaji: Delay-dependent stabilization conditions of controlled positive T-S fuzzy systems with time varying delay. Int. J. of Innovative Computing, Information and Control, 7 (2011), 1533-1548.

[2] A. Ben Abdallah, I. Ellouze and M.A. Hammami: Practical stability of nonlinear time-varying cascade systems. J. of Dynamical and Control Systems, 15 (2009), 45-62.

[3] A.G. Soldatos and M. Corless: Stabilizing uncertain systems with bounded control. Dynamics and Control, 1 (1991), 227-238.

[4] Bassem Ben Hamed, Imen Ellouze and M.A. Hammami: Practical uniform stability of nonlinear differential delay equations. Mediterranean J. of Mathematics, 6 (2010), 139-150.

[5] Bassem Ben Hamed and M.A. Hammami: Practical stabilisation of a class of uncertain time-varying nonlinear delay systems. J. of Control Theory and Applications, 7 (2009), 175-180.

[6] S.G. CAO, N.W. RESS and G. FENG: Stability analysis and design for class of continuous-time fuzzy control systems. Int. J. of Control, 64 (1996), 1069-1087.

[7] G. Feng, S.G. GaO, N.W. Ress and G.K. Chack: Design of fuzzy control systems with guaranteed stability. Fuzzy Sets Systems, 85 (1997), 1-10.

[8] J. PARK, J. KIM and D. PARK: LMI-based design of stabilizing fuzzy controllers for nonlinear systems described by Takagi-Sugeno fuzzy model. Fuzzy Sets Systems, 122 (2003), 73-82. 
[9] K. TANAKA, T. IKEDA and H.O. WANG: Robust stabilization of a class of uncertain nonlinear systems via fuzzy control: quadratic stabilizability, $H_{\infty}$ control theory and linear matrix inequalities. IEEE Trans. Fuzzy Systems, 4 (1996), 1-13.

[10] Ligang Wu, Xiaojie Su, Peng Shi and Jianbin Qiu: A new approach to stability analysis and stabilization of discrete-time T-S fuzzy time-varying delay systems. IEEE Trans. on Systems, Man, and Cybernetics- Part B: Cybernetics, 41 (2011), 273-286.

[11] Ligang Wu, Xiaojie Su, Peng Shi and Jianbin QiU: Model approximation for discrete-time state-delay systems in the $\mathrm{T}-\mathrm{S}$ fuzzy framework. IEEE Trans. Fuzzy Systems, 19 (2011), 366-378.

[12] V. Lakshmikantham, S. Leela and A.A. Martynnyuk: Practical Stability of Nonlinear Systems. World Scientific Singapore, 1990.

[13] J.P. LASAlLE and S. LefSChETZ: Stability by Lyapunov's Direct Method with Application. Academic Press New York, 1961.

[14] Linna Zhou, Qingling Zhang and Chunyu YANG: Practical stability analysis and synthesis of a class of uncertain T-S fuzzy systems. Fuzzy Systems and Knowledge Discovery Lecture Notes in Computer Science, 4223 (2006), 11-20.

[15] LIU, ZHANG: New approach to $H_{\infty}$ controller designs based on fuzzy observers for T-S fuzzy systems via LMI. Automatica, 39 (2003), 1571-1582.

[16] M. CORLESS and G. LEITMANN: Continuous state feedback guaranteeing uniform ultimate boundedness for uncertain dynamic systems. IEEE Trans. on Automatic Control, 26 (1981), 1139-1143.

[17] M. CORLESS: Guaranteed rates of exponential convergence for uncertain systems. J. of Optimization Theory and Applications, 64 (1990), 481-494.

[18] M. Corless and G. Leitmann: Bounded controllers for robust exponential convergence. J. of Optimization Theory and Applications, 76 (1993), 1-12.

[19] N. Hadj Taieb, M.A. Hammami, F. Delmotte and M. Ksontini: On the global stabilization of Takagi-Sugeno fuzzy cascaded systems. Nonlinear Dynamics, 67 (2012), 2847-2856.

[20] M. Sugeno and G.T. Kang: Structure identification of fuzzy model. Fuzzy Sets Systems, 28 (1988), 15-33.

[21] TAI-ZU WU and YAU-TARNG JUANG: Design of variable structure control for fuzzy nonlinear systems. Expert System with Applications, 35 (2008), 1496-1503. 
[22] T. TAKagi and M. Sugeno: Fuzzy identification of systems and its applications to modeling and control. IEEE Trans. on Systems, Man, and Cybernetics- Part B: Cybernetics, 15 (1985), 116-132.

[23] R.M. Tong: A control engineering review of fuzzy systems. Automatica, 13 (1977), 559-568.

[24] XiaO-Heng Chang and Guang-Hong Yang: Relaxed results on stabilization and state feedback $H_{\infty}$ control conditions for T-S fuzzy systems. Int. J. of Innovative Computing, Information and Control, 7 (2011), 1753-1764.

[25] Xiaojie Su, Peng Shi, Ligang Wu and Yong-Duan Song: A novel approach to filter design for T-S fuzzy discrete-time systems with time-varying delay. to appear in IEEE Transactions Fuzzy Systems.

[26] H.O. WAng, K. TANAKA and M. GRIFFIn: Parallel distributed compensation of nonlinear systems by Takagi and Sugeno's model. Proceedings of Fuzzy'95, (1995).

[27] H.O. WANG, K. TANAKA and M. GRIFFIN: An approach to fuzzy control of nonlinear systems: Stability and design issues. IEEE Trans. Fuzzy Systems, 4 (1996), $14-23$.

[28] W. TAng, G. CHEN and R. LU: A modified fuzzy PI controller for a flexible-joint robot arm with uncertainties. Fuzzy Sets Systems, 118 (2001), 109-119.

[29] Wen-Jer Chang, Cheung-Chieh Ku and PeI-Hwa Huang: Robust fuzzy control via observer feedback for passive stochastic fuzzy systems with time-delay and multiplicative noise. Int. J. of Innovative Computing, Information and Control, 7 (2011), 345-364.

[30] L.A. ZADEH: Outline of a new approach to the analysis of complex systems and decision process. IEEE Trans. on Systems, Man, and Cybernetics- Part B: Cybernetics, 3 (1973), 28-44.

[31] J.M. ZHANG, R.H. Li and P.A. ZHANG: Stability analysis and systtematic design of fuzzy control systems. Fuzzy Sets Systems, 120 (2001), 65-72.

[32] Z.J. Wu, X.J. XIE and S.Y. ZHANG: Stochastic adaptive backstepping controller design by introducing dynamic signal and changing supply function. Int. J. of Control, 79 (2006), 1635-1646.

[33] Z.P. JiAng and L. PRALY: Design of robust adaptive controllers for nonlinear systems with dynamic uncertainties. Automatica, 34 (1998), 825-840. 